Z. klin. Chem. u. klin. Biochem.

8. Jg., S. $534-536$, September 1970

\title{
Eine fluorometrische Methode zur Bestimmung des Progesterons im Plasma
}

\author{
Von V. Graef, E. Nowotny ${ }^{1}$ ) und HJ. Staudinger \\ Aus dem Biochemischen Institut der Universität Gießen (Direktor: Prof. Dr. Hj. Staudinger)
}

(Eingegangen am 22. Juni 1970)

Es wird eine Methode zur Bestimmung des Progesterons im Plasma von Frauen während des Zyklus beschrieben. Das Progesteron wird durch eindimensionale Dünnschichtchromatographie abgetrennt und durch die Fluoreszenzreaktion auf der Oberfläche von Lithiumhydroxid quantitatir bestimmt. Die Bestimmung läßt sich schnell ausführen. Bei Verwendung von $10 \mathrm{~m} l$ Plasma wird eine Empfindlichkeit von $0,1 \mu \mathrm{g} / 100 \mathrm{~m} l$ erreicht.

\section{A fluorometric method for the determination of progesterone in plasma}

A method for the determination of progesterone in the plasma of women during the ovulatory cycle is described. Progesterone is separated by one-dimensional thin-layer chromatography and determined quantitatively by the fluorescence reaction on the surface of lithium hydroxide. The method is rapid. The sensitivity is $0.1 \mu \mathrm{g} / 100 \mathrm{~m} l$ when $10 \mathrm{~m} l$ plasma are used.

Zur Bestimmung des Progesterons im Plasma werden heute vorwiegend gaschromatographische $(1,2,3)$ und Doppelisotopenverdünnungsmethoden $(4,5)$ benutzt. Diese Methoden sind zwar sehr empfindlich und spezifisch, doch benötigen sie kostspielige Geräte, die vorläufig für routinemäßige Untersuchungen nur selten zur Verfügung stehen. Deshalb haben wir eine früher in unserem Institut entwickelte Methode (6) zur fluorometrischen Bestimmung von $\Delta^{4}-3$-Oxosteroiden zur quantitativen Bestimmung des Progesterons im Plasma herangezogen. Diese Methode beruht darauf, daß $\triangle^{4}$-3-Oxosteroide auf der Oberfläche eines Lithiumhydroxid-Preßlings eine spezifische Fluoreszenz erzeugen. Dieses Prinzip wurde bereits zur Bestimmung des Aldosterons im Harn (7) und des Testosterons im Harn (8) und im Plasma (9) angewandt. Die Empfindlichkeit der Methode reicht aus, um die Schwankungen des Progesterongehaltes des Plasmas während des normalen Zyklus erfassen zu können.

\section{Methodik}

Reagenzien

Diätbyläther: DAB 6 (Firma Riedel de Haen) wird über Natriumdraht destilliert. Er wird in einer braunen Flasche aufbewahrt und soll nicht älter als 3 Tage sein.

Metbylenchlorid: 2 l Methylenchlorid werden mit $200 \mathrm{ml} 0,1$ proz. Kaliumpermanganat-Lösung, mit $200 \mathrm{~m} / 1 \mathrm{~N} \mathrm{NaOH}$ und dreimal mit je $400 \mathrm{~m} l$ Wasser ausgeschüttelt. Nach 24stdg. Trocknen über wasserfreiem Calciumchlorid wird das Lösungsmittel destilliert.

Chloroform: p. a. (Firma E. Merck, Darmstadt)

Benzol: p. a. (Firma E. Merck, Darmstadt)

Äthylacetat: p. a. (Firma E. Merck, Darmstadt)

n-Hexan: rein (Firma E. Merck, Darmstadt)

Metbanol: p. a. (Firma E. Merck, Darmstadt) wird über Natriumborhydrid $(2 \mathrm{~g} / l)$ destilliert und dann an einer Füllkörperkolonne redestilliert.

1) Stipendiat der Alexander-von-Humboldt-Stiftung.
Aluminiumoxid: $\mathrm{GF}_{254}$ für Dünnschichtchromatographie (Firma E. Merck, Darmstadt)

Dünnschichtplatten: Glasplatten $(20 \times 20 \mathrm{~cm})$ werden mit einer $0,25 \mathrm{~mm}$ dicken Schicht Aluminiumoxid $\mathrm{GF}_{254}$ versehen, $3 \mathrm{Stdn}$. im Trockenschrank bei $110^{\circ}$ erhitzt und in einem Exsikkator ohne Trockenmittel aufbewahrt.

Litbiumbydroxid: etwa 98\% (Firma E. Merck, Darmstadt)

Progesteron: (Firma E. Merck, Darmstadt).

Die verwendeten Glasgeräte werden mit Chromschwefelsäure gereinigt und sehr gründlich mit Leitungswasser und dest. Wasser gespült.

Extraktion des Plasmas

Blut wird unter Zusatz von Heparin $(0,2 \mathrm{ml}=200 \mathrm{E} / 20 \mathrm{~m} l \mathrm{Blut})$ abgenommen und sofort zentrifugiert. $10 \mathrm{~m} l$ Plasma werden mit $3 \mathrm{~m} l 1 \mathrm{~N} \mathrm{NaOH}$ versetzt und einmal mit $50 \mathrm{~m} l$ und einmal mit $30 \mathrm{ml}$ absol. Äther extrahiert. Man wäscht die vereinigten Ätherextrakte zweimal mit je $10 \mathrm{ml}$ dest. Wasser, trocknet sie über wasserfreiem Natriumsulfat und verdampft den Äther am Rotationsverdampfer. Den Rückstand versetzt man mit $7 \mathrm{~m} /$ Methanol und $3 \mathrm{ml}$ Wasser, überführt die Lösung nach Abkühlen in einen Schütteltrichter und spült mit $5 \mathrm{ml} 70$ proz. Methanol nach. Die wäßrig-methanolische Lösung wird mit $5 \mathrm{ml} n$-Hexan ausgeschüttelt. Nach kurzem Zentrifugieren entfernt man die obere Phase und dampft die wäßrig-methanolische Lösurig am Rotationsverdampfer bei $40^{\circ}$ ein. Reste von Wasser werden durch Abdampfen mit Äthanol entfernt. Der Verdampfungsrückstand wird mit dreimal je $2 \mathrm{~m} l$ Chloroform in ein spitzes Zentrifugenröhrchen überführt, die Lösung dampft man am Rotationsverdampfer ein.

Dünnschichtchromatographie

Man trägt den Verdampfungsrückstand des Plasmaextraktes mit. dreimal $100 \mu l$ Chloroform auf eine Dünnschichtplatte (Aluminiumoxid $\left.\mathrm{GF}_{254}\right) 2 \mathrm{~cm}$ vom unteren Rand entfernt auf. Links und rechts neben dem Plasmaextrakt werden als Bezugssubstanz je $3 \mu \mathrm{g}$ Progesteron in $10 \mu \mathrm{l}$ Chloroform aufgetragen. Man entwickelt die Platte im System Benzol/Methylenchlorid/Äthylacetat $(3: 1: 1 \mathrm{~V} / \mathrm{V})$ und markiert die Progesteron enthaltende Zone des Plasmaextraktes anhand der beiden unter der UVLampe erkennbaren Progesteron-Flecken. Die Zone mit dem Progesteron des Plasmaextraktes wird ausgekratzt, das Aluminiumoxid wird in $2 \mathrm{~m} l$ Methanol aufgenommen und unter Zusatz einer Glasperle 5 Min. in einer Schüttelmaschine geschüttelt. Dann zentrifugiert man bei $3000 \mathrm{U}$./Min. und pipettiert das Eluat in ein spitzes Zentrifugenröhrchen, in dem es einge- 
dampft wird. Das Aluminiumoxid wird nochmal in der gleichen Weise mit $2 \mathrm{~m} /$ und dann mit $1 \mathrm{ml}$ Methanol eluiert. Die Eluate werden im gleichen Spitzröhrchen eingedampft. Zur Gewinnung eines „Plattenleerwertes" kratzt man eine Fläche, die dem PlasmaProgesteron-Fleck in der Größe entspricht, in Höhe des Progesterons von einer Stelle der Platte, wo kein Steroid gelaufen war. Das Adsorbens wird in der gleichen Weise eluiert.

\section{Quantitative Bestimmung}

Die Lithiumhydroxid-Preßlinge werden in der schon beschriebenen Weise (6) hergestellt. Man löst den Verdampfungsrückstand der Eluate vom Hauptwert (Plasma-Progesteron) und vom Plattenleerwert in $100 \mu /$ Chloroform und engt die Lösung durch Einblasen von Stickstoff auf etwa $10 \mu l$ ein. Diese Lösung trägt man auf den Lithiumhydroxid-Preßling auf. Das Spitzröhrchen wird noch zweimal mit je $100 \mu l$ Chloroform ausgespült. Diese Lösungen werden nach Einengen auf $10 \mu /$ auf den gleichen Preßling aufgetragen. Zur Bestimmung eines Standard-Wertes engt man eine Lösung von $0,1 \mu \mathrm{g}$ Progesteron/100 $\mu \mathrm{l}$ Chloroform in einem Spitzröhrchen mit Stickstoff auf $10 \mu l$ ein und trägt diese Lösung auf einen Lithiumhydroxid-Preßling auf. Das Röhrchen wird in der gleichen Weise wie beim Hauptwert ausgespült. Zur Bestimmung eines Reagenzien-Leerwertes engt man $100 \mu /$ Chloroform im Spitzröhrchen auf $10 \mu /$ ein, trägt diese auf einen Preßling auf und spült dieses Röhrchen ebenfalls noch zweimal mit je $100 \mu l$ Chloroform aus. Die Fluoreszenz der Preßlinge wird vor und nach $20 \mathrm{Min}$. Erhitzen auf $100^{\circ}$ (Trockenschrank) in einem Photometer "Eppendorf" mit Fluoreszenzzusatz gemessen. Die während des Erhitzens auftretende Fluoreszenz ist der Progesteronmenge proportional. Die Fluoreszenz des Hauptwertes wird in folgender Weise errechnet:

$F_{H}=F_{H(\text { nach })}-F_{H}$ (vor) $\quad H=$ Hauptwert

$F_{P L}=F_{P L}$ (nach) $-F_{P L}$ (vor) $\quad P L=$ Plattenleerwert

$F_{P P}=F_{H}-F_{P L} \quad F=$ relative Intensität der Fluoreszenz

(vor) $=$ vor dem Erhitzen

$($ nach $)=$ nach dem Erhitzen

$\mathrm{PP}=$ Plasma-Progesteron

Die Fluoreszenz für den Progesteron-Standard errechnet man in folgender Weise:

$$
\begin{aligned}
& F_{S}=F_{S \text { (nach) }}-F_{S} \text { (vor) } \\
& \mathrm{F}_{\mathrm{RL}}=\mathrm{F}_{\mathrm{RL} \text { (nach) }}-\dot{\mathrm{F}}_{\mathrm{RL} \text { (vor) }} \\
& \mathrm{RL}=\text { Reagenzien-Leerwert } \\
& \mathrm{S}=\text { Standard }
\end{aligned}
$$

$F_{\text {Stand.-P }}=F_{S}-F_{R I}$

In der untersuchten Probe sind $\frac{0,1 \cdot F_{P P}}{F_{\text {Stand.-P }}} \mu \mathrm{g}$ Progesteron enthalten.

\section{Ergebnisse}

\section{Spezifität der Methode}

Wie in früheren Untersuchüngen (10) festgestellt wurde, ist die Fluoreszenzreaktion auf Lithiumhydroxid-Preßlingen spezifisch für $\Delta^{4}-3-O x$ xosteroide. Wie aus den Angaben in Tabelle 1 ersichtlich ist, ist Progesteron

Tab. 1

\begin{tabular}{|c|c|}
\hline Steroid & $\mathbf{R F}_{\mathbf{F}}$ \\
\hline Cortisol & 0 \\
\hline Corticosteron & 0 \\
\hline $11 \beta$-Hydroxy-progesteron & 0,15 \\
\hline $20 \alpha$-Hydroxy-progesteron & 0,23 \\
\hline Adrenosteron & 0,28 \\
\hline 20 $\beta$-Hydroxy-progesteron & 0,29 \\
\hline 11-Oxo-progesteron & 0,38 \\
\hline Androstendion- $(3,17)$ & $0,5.6$ \\
\hline Progesteron & 0,67 \\
\hline
\end{tabular}

$R_{\mathrm{F}}$-Werte verschiedener $\Delta^{3}-3-$ Oxosteroide, Dünnschichtchromato-

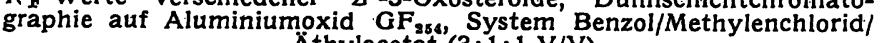
Âthylacetat' $(3: 1: 1 \mathrm{~V} / \mathrm{V})$ bei der Dünnschichtchromatographie von anderen $\Delta^{4}$-3-Oxosteroiden getrennt. Wir untersuchten ferner Sammelplasma von Männern nach der angegebenen Methode. Der Progesterongehalt dieses Plasmas lag unter der Nachweisgrenze der Methode $(0,1 \mu \mathrm{g} / 100 \mathrm{ml})$.

\section{Richtigkeit der Methode}

Zur Prüfung der Richtigkeit der Methode wurden je $10 \mathrm{ml}$ Sammelplasma von Männern 0,025 und 0,05 $\mu \mathrm{g}$ Progesteron zugesetzt, von denen 96 bzw. $90 \%$ wiedergefunden wurden (Tab. 2).

Tab. 2

Ergebnisse von Wiederfindungsversuchen, bei denen jeweils $10 \mathrm{~m} l$ Männerplasma verschiedene Mengen Progesteron zugesetzt wurden

\begin{tabular}{cc}
$\begin{array}{c}\text { zugesetzt } \\
\mu \mathrm{g}\end{array}$ & Progesteron \\
\hline 0,025 & $\begin{array}{c}\mu \mathrm{g} \\
\text { wiedergefunden }\end{array}$ \\
0,025 & 0,026 \\
0,025 & 0,024 \\
0,025 & 0,021 \\
0,025 & 0,024 \\
& 0,027 \\
0,05 & $\overline{\mathbf{x}}=0,024(96 \%)$ \\
0,05 & 0,048 \\
0,05 & 0,038 \\
0,05 & 0,048 \\
0,05 & 0,049 \\
0,05 & 0,048 \\
& 0,045 \\
\hline
\end{tabular}

\section{Genauigkeit der Methode}

Der Progesterongehalt eines Mischplasmas wurde zehnmal bestimmt. Dabei wurde als Mittel eine Konzentration von $0,76 \mu \mathrm{g} / 100 \mathrm{ml}$ gefunden. Die StandardAbweichung betrug $\mathrm{s}=0,037 \mu \mathrm{g} / 100 \mathrm{ml}$.

Tab. 3

Genauigkeit der Methode. Mehrfachbestimmung von Mischplasma

\begin{tabular}{c}
$\begin{array}{c}\text { Progesteron } \\
\mu \mathrm{g} / 100 \mathrm{ml}\end{array}$ \\
0,73 \\
0,79 \\
0,71 \\
0,76 \\
0,81 \\
0,71 \\
0,77 \\
0,79 \\
0,81 \\
0,76 \\
\hline $\bar{x}=0,76$ \\
Standard-Abweichung s $=0,037$ \\
Variationskoeffizient $\mathrm{V}=4,9 \%$ \\
\hline
\end{tabular}

\section{Empfindlichkeit}

Mit der angegebenen Methode ist es möglich, Progesteron-Konzentrationen bis $0,1 \mu \mathrm{g} / 100 \mathrm{ml}$ Plasma $\mathrm{zu}$ bestimmen.

\section{Normalwerte}

Wir bestimmten den Progesteron-Gehalt des Plasmas von 7 Frauen während der lutealen Phase und fanden 
eine durchschnittliche Progesteron-Konzentration von $1,38 \mu \mathrm{g} / 100 \mathrm{~m} l$. In der präovulatorischen Phase wurden 0,20 bzw. $<0,10 \mu \mathrm{g} / 100 \mathrm{~m} l$ bestimmt ('Tab. 4).

Tab. 4

Progesteron im Plasma von Frauen während des normalen Zyklus

\begin{tabular}{|c|c|}
\hline Zyklustag & Progesteron $(\mu \mathrm{g} / 100 \mathrm{ml})$ \\
\hline \multicolumn{2}{|c|}{ Luteale Phase } \\
\hline 20 & 1,00 \\
\hline 20 & 1,02 \\
\hline 19 & 1,23 \\
\hline 20 & 1,61 \\
\hline 18 & 1,95 \\
\hline 16 & 1,05 \\
\hline \multirow[t]{2}{*}{$12 *)$} & 1,84 \\
\hline & $\bar{x}=1,38$ \\
\hline \multicolumn{2}{|c|}{ Präovulatorische Phase } \\
\hline 5 & 0,20 \\
\hline 5 & $<0,10$ \\
\hline
\end{tabular}

*) Zyklus von 21 Tagen

\section{Diskussion}

Die hier beschriebene Methode eignet sich zur schnellen und empfindlichen Bestimmung des Progesterons im Plasma vọn Frauen. Sie läßt sich mit einfachen Mitteln ohne Verwendung von isotopenmarkierten Verbindungen oder eines Gaschromatographen ausführen. Die
Lipoide werden in üblicher Weise durch Verteilung $z$ wischen 70 proz. Methanol und $n$-Hexan entfernt. In der folgenden eindimensionalen Dünnschichtchromatographie wird Progesteron von anderen $\Delta^{4}$-3-Oxosteroiden getrennt, denn nur diese geben die Fluoreszenzreaktion auf der Oberfläche von LithiumhydroxidPreßlingen. Aufgrund der weenigen Reinigungsschritte beträgt die Wiederfindung $90-96 \%$. Wegen des geringen Aufwandes kann diese Methode zur toutinemäßigen Bestimmung des Progesterons im Plasma von Frauen verwendet werden. In Männerplasma wurde erwartungsgemä $\beta$ eine Progesteron-Konzentration von weniger als $0,1 \mu \mathrm{g} / 100 \mathrm{ml}$ gefunden. Nach den Angaben in der Literatur $(1,4,5)$ wurden im Plasma von Männern durchschnittlich $0,03 \mu \mathrm{g}$ Progesteron $/ 100 \mathrm{ml}$ gefunden. Die von uns im Plasma von Frauen während des Zyklus gefundenen Progesteronmengen stimmen mit den Ergebnissen anderer Autoren überein. Die Mittelwerte der Progesteron-Konzentration im Plasma in der lutealen Phase liegen bei anderen Untersuchern $z$ wischen 1,04 und $1,52 \mu \mathrm{g} / 100 \mathrm{ml}$, während wir als Mittelwert 1,38 $\mu \mathrm{g} / 100 \mathrm{~m} l$ gefunden haben. Die Empfindlichkeit von $0,1 \mu \mathrm{g} / 100 \mathrm{ml}$ reicht aus, um Schwankungen des Progesteron-Gehaltes des Plasmas während des Zyklus erfassen zu können.

\section{Literatur}

1. Van der Molen, H. J. und D. Groen, J. Clin. Endoct. Springfield 25, 1625 (1965). - 2. WYMAN, H. und I. F. SOMMERville, Steroids 12, 63 (1968). - 3. ZaNDER, J. und B. RunNeBAUM in R. I. DorfmaN (Ed.) Methods in Hormone Research, Vol I, S. 212, Academic Press, New York-London (1968). 4. Rrondel, A., J. F. TAIt, S. A. S. TaIt, M. Gut und B. LitTile, J. Clin. Endocr., Springfield 25, 229 (1965). - 5. Yoshimr, T. und M. B. LIPSETT, Steroids 11, 527 (1968). - 6. NowotNY, E., R. Abrahiam und HJ. Staudinger, diese Z. 3, 8 (1965). - 7. Nowotny, E. und HJ. Staudinger, diese Z. 4, 203 (1966). 8. Graef, V., P. Jobst und HJ. Staudinger, diese $Z$. 6,159 (1968). - 9. Graef, V. und HJ. Staudinger, diese Z. 6, 280 (1968). - 10. Abraham, R. und HJ. Staudinger, Z. Naturforsch. 18b, 421 (1963).
Prof. Dr. Hj. Staudinger 6300 Gießen

Friedrichstr. 24 